Cinémas

Revue d'études cinématographiques

Journal of Film Studies

\title{
Top Hat et l'intermédialité de la comédie musicale
}

\section{Jürgen E. Müller}

Volume 5, numéro 1-2, automne 1994

URI : https://id.erudit.org/iderudit/1001014ar

DOI : https://doi.org/10.7202/1001014ar

Aller au sommaire du numéro

Éditeur(s)

Cinémas

ISSN

1181-6945 (imprimé)

1705-6500 (numérique)

Découvrir la revue

Citer cet article

Müller, J. E. (1994). Top Hat et l'intermédialité de la comédie musicale. Cinémas, 5(1-2), 211-220. https://doi.org/10.7202/1001014ar

\section{Résumé de l'article}

La comédie musicale Top Hat de Mark Sandrich (1935) constitue l'un des plus grands succès cinématographiques aux États-Unis. Si l'utilisation des conventions du genre et la présence des normes sociales de la société américaine ont contribué au succès du film, l'auteur démontre ici que les structures médiatiques du film ont aussi joué un rôle déterminant dans la réception du film, analysant ce travail à partir des concepts de médialité et d'intermédialité. 


\title{
Top Hat et l'intermédialité de la comédie musicale ${ }^{1}$
}

\section{Jürgen E. Müller}

\begin{abstract}
RÉSUMÉ
La comédie musicale Top Hat de mark Sandrich (1935) constitue l'un des plus grands succès cinématographiquesaux États-Unis. Si l'utilisation des conventions du genre et la présence des normes sociales de la société américaine ont contribué au succès du film, l'auteur démontre ici que les structures médiatiques du film ont aussi joué un rôle déterminant dans la réception du film, analysant ce travail à partir des concepts de médialité et d'intermédialité.
\end{abstract}

\section{ABSTRACT}

The musical comedy Top Hat by Mark Sandrich (1935) was one of the most successful films in United States. While the use of genre conventions and the presence of American social norms undoubtedly contributed to its success, the author of this text shows that the film's media structures also played a determining role in the film's reception, analyzing the work from the point of view of its mediality and its intermediality.

\section{Top Hat et comédie musicale}

Dès ses débuts, la comédie musicale constitue un genre multimédiatique et intermédiatique par excellence. C'est la fascination du mot parlé et chanté, de la musique, des corps en mouvement, de la danse, des concepts du théâtre, de l'opéra, de l'opérette, de la peinture... qui, parmi d'autres facteurs, fait que ce genre filmique subsiste depuis plus de soixante-dix ans. La comédie musicale leurre le spectateur et lui offre, à travers ses 
structures dichotomiques (en ce qui concerne, par exemple, la représentation des relations entre "femmes et hommes") un spectacle évasif et rédemptif qui se place dans un contexte historique de conventions et de valeurs d'une société.

Dans l'histoire du genre et de sa réception, Top Hat de Mark Sandrich (1935) marque un des points culminants ${ }^{2}$. Cette comédie musicale, qui peut être caractérisée comme "Fairy Tale Musical" (Altman, p. 129), a été un des plus grands succès aux États-Unis. En ce qui concerne les raisons possibles de ce succès extraordinaire, il faudra les chercher dans une interaction très différenciée entre les conventions du genre et les normes sociales de la société américaine ${ }^{3}$. Mais — en dehors des deux vedettes de ce film, Fred Astaire et Ginger Rodgers - les structures médiatiques ont aussi joué un rôle important dans la réception de cette comédie musicale. C'est le spectacle médiatique qui attire l'intérêt du spectateur.

\section{Médialité de la comédie musicale}

Si je parle de la médialité ${ }^{4}$ de la comédie musicale, c'est que je pars de l'idée d'un assemblage, d'un labyrinthe, d'une interaction médiatiques qui caractérisent la structure médiatique de ce genre et qui forment des facteurs principaux par rapport à la fascination vécue par le spectateur regardant le "texte filmique". Comme Jean-François Lyotard l'a décrit :

L'importance d'un texte n'est pas sa signification, ce qu'il veut dire, mais ce qu'il fait et fait faire. Ce qu'il fait: la charge en affects qu'il détient et communique; ce qu'il fait faire: les métamorphoses de cette énergie potentielle en d'autres choses: d'autres textes, mais aussi de peintures, photographies, séquences de film, actions politiques, décisions, inspirations érotiques, refus d'obéir, initiatives économiques (p. 6).

Les énergies des textes médiatiques basculent dans autre chose, dans d'autres textes, d'autres actions. Pour Lyotard, les transformations médiatiques sont un élément important pour éviter une immobilité qui figerait les significations du texte. Le jeu intermédiatique accélère le "vertige des signifiants" alors que ses contingences garantissent la dissociation et l'hétérogénéités. 
Si nous entendons par "intermédialité ${ }^{6}$ " qu'il y a des relations médiatiques variables entre les médias et que leur fonction naît entre autres de l'évolution historique de ces relations ${ }^{7}$, cela implique alors que la conception de "monades" ou de sortes de médias "isolés" est irrecevable (ce qui ne signifie pas pour autant que les médias se plagient mutuellement, mais qu'au contraire, ils intègrent à leur propre contexte des questions, des concepts, des principes qui se sont développés au cours de l'histoire de l'art figuratif et sonore occidental ${ }^{8}$ ). Un film devient inter médiatique quand il transpose le "côte à côte" multimédiatique de citations médiatiques, en complicité sur le plan de la conception, et lorsque les ruptures et stratifications esthétiques permettent d'autres dimensions à l'expérience et au vécu. C'est alors dans la reconstruction de relations intermédiatiques que l'on trouve l'un des centres d'intérêt de l'histoire des médias et de la sémiologie.

Mais revenons à Top Hat. Quels éléments constituent le jeu et le labyrinthe intermédiatique de ce film et comment pourrait-on décrire la "spécificité médiatique " de cette comédie musicale?

\section{Quelques remarques sur l'intermédialité de Top Hat}

- RAdio Pictures et COMÉdie musicale

Le générique du film nous apprend que Top Hat a été produit par les studios Radio Pictures. Le nom de ces studios fut donné dans la première phase du parlant et il nous renvoie à un aspect très important de l'intermédialité du film. Avec l'introduction du médium "son" dans le film (qui, à cette période, n'était naturellement pas si muet que l'on aime toujours le penser) se posait la grande question de savoir quelle pourrait être le nom adéquat de ce nouvel hybride médiatique qui a commencé à inclure le médium acoustique, la bande-son. Et ce n'est pas un hasard si parmi un groupe de définitions concurrentielles de la nouvelle qualité médiatique du "film parlant ", le nom de Radio Pictures a pu devenir une des dénominations préférées. La radio ${ }^{9}$ et ses structures médiatiques ont en effet eu une influence considérable sur le développement du parlant ${ }^{10}$.

En 1935, les structures médiatiques et esthétiques du parlant paraissent stabilisées, après une phase plus au moins expérimen- 
tale au cours des deux ou trois premières années du parlant, mais le nom Radio Pictures est toujours un indice de la recherche d'une (nouvelle) identité du médium «film». Et nous trouvons donc aussi, dans Top Hat, quelques traces de cette influence (par exemple, dans les scènes de communication médiatisée par des appareils modernes, comme le téléphone et le télégraphe).

\section{— LE LABYRINTHE MÉdiATIQUE DE TOP HAT}

C'est dans la nature de la chose, ou plutôt du genre, que Top Hat nous offre un éventail de re-présentations médiatiques. Le genre joue avec la fascination du spectateur qui résulte non seulement de la transgression des conventions du permis et de l'interdit, mais qui résulte aussi du spectacle que signifie la présence et l'interaction d'un grand nombre de médias anciens et modernes. Je vais nommer les plus évidents, relatifs d'abord à la re-présentation du médium expressif par excellence:

- Le corps humain qui devient littéralement un corps sonore et un corps métaphorique. C'est un corps qui bouge et qui fait du bruit ${ }^{11}$. (Mais le film montre aussi les spectateurs privés et publics de ses corps sonores humains. Il y a plusieurs exemples de cette "archi "-scène de la production et de la réception médiatiques: quelqu'un chante ou danse et est regardé par un public charmé ou enthousiasmé.)

- La danse avec ou sans claquettes.

- La danse parlée, la musique et les effets de son.

- L'intégration parfois presque obsessive du téléphone et du télégraphe dans le cadre filmique.

- L'utilisation de structures médiatiques propres à l'opéra, à l'opérette et au théâtre.

- Le recours à des sujets et à des modalités de représentation empruntées à la peinture.

Toutes ces structures ou citations médiatiques sont intégrées dans le contexte de notre film, elles sont ré-arrangées dans un réseau dynamique d'imbrications médiatiques. Le film veut faire voir et entendre son intermédialité, il joue avec les conventions médiatiques de ce qui peut être montré d'après les attentes de son public. La fascination exercée sur le spectateur résulte de ce 
jeu de la représentation de différentes options médiatiques et de l'interférence (moderne?) de ces options. Même si Top Hat n'est pas aussi autoréflexif ${ }^{12}$ par rapport à ses structures médiatiques que les premiers films parlants, il y a dans le film une tendance assez marquée à l'autoréflexivité médiatique. Mais, bien sûr, par rapport aux nouveaux médias, la comédie musicale se caractérise par son effort de synchronisation de différents éléments et structures médiatiques, ce qui la distingue précisément de la plupart des productions postmodernes des nouveaux médias, par exemple de l'art vidéo. Là, cette synchronisation standard caractéristique d'Hollywood n'est plus recherchée.

\section{- ThaCKERY CLUB - UNE OUVERTURE INTERMÉdiatiQue}

Revenons au caractère intermédiatique et autoréflexif de notre film. J'aimerais diriger mon attention sur la séquence d'ouverture, au moment où on pénètre au Thackery Club, une séquence qui fut analysée d'une manière très lucide par Rick Altman ${ }^{13}$.

"Silence", stipule la pancarte - et ce silence fait surgir un fort désir du spectateur: "[...] la comédie musicale et le silence ne sauraient cohabiter et bien vite l'on se met à attendre si ce n'est une chanson, au moins un mot, un signe de vie. C'est à une rafale de claquettes que l'on a droit [... $]^{14}$ " (Altman, p. 176).

Ce sont les structures du film muet (sans orchestre ni présentateur), ce sont les images forcées d'être muettes qui renvoient le spectateur aux possibilités intermédiatiques du film parlant. Il lui est interdit d'entendre des sons, mais il veut entendre des sons. Ce jeu autoréflexif du médium n'est qu'une autre forme d'accentuation de la dimension sonore. Dans un grand nombre des premiers films parlants, cette dimension s'est fait entendre par des voix venant d'une étoile sombre au début du film. C'était la fascination des génériques parlés dans une salle de projection sombre. Mais dans la première séquence de Top Hat, on voit des personnes sans pouvoir les entendre. Les sons entrent dans cet univers muet d'une manière presque radiophonique, ou plutôt, à la manière d'une pièce radiophonique. Ce sont les détails sonores banals et généralement pas inaperçus, le tintement des verres, la toux, le bruissement d'un journal, qui réjouissent 
le spectateur et qui le confortent dans ses attentes et dans sa connaissance des conventions du genre. L'explosion finale des claquettes de Fred Astaire le replace dans son horizon d'attente par rapport aux structures intermédiatiques du genre comédie musicale. (Cette procédure me semble appuyer la thèse du caractère radiophonique des Radio Pictures.) Ainsi la scène d'ouverture nous renvoie au caractère médiatique du genre et aux attentes correspondantes du spectateur. Un déficit par rapport à la présence du médium acoustique fait sentir la fonction habituelle du son dans le Hollywood musical.

\section{- No StRINGS ET L'ESPACE INTERMÉdIATIQUE}

ET MÉTAPHORIQUE DE LA COMÉDIE MUSICALE

Sur la toile de fond de notre perspective analytique de l'intermédialité, la représentation de la première rencontre entre Fred et Ginger (qui a lieu dans la chambre d'hôtel de l'ami de Fred et qui est nécessaire selon les conventions du genre, mais problématique selon les conventions de la société américaine - une dame ne rend pas visite à un homme inconnu, et surtout pas pendant la nuit) gagne une nouvelle qualité. Le film construit un espace sonore métaphorique où l'histoire d'amour pourra se dérouler. Rappelons-nous que Fred pratique ses claquettes dans la chambre au-dessus de la chambre de Ginger et que cette sorte de courtship behavior non intentionnel, cette façon de danser lui gagnera enfin le cœur même de Ginger (pour nous rappeler du titre allemand de notre film: Ich tanze mich in dein Herz hinein).

Comment cette danse se trouve-t-elle représentée? Il y a, comme je viens de le mentionner, cette sorte $d$ ' "archi "-situation du film parlant, où un spectateur est intégré dans la scène du spectacle. Dans ce cas-là, c'est l'ami anglais du danseur américain. La réception (fictive) du spectacle fait partie intégrale de sa représentation.

Mais, dans cette scène, une autre sorte de réception est également montrée au spectateur du film. C'est la réception du bruit encore "gênant" par Ginger, qui se trouve dans son lit dans la chambre juste au-dessous de celle de Fred. Un mouvement/ trajet de la caméra lie les deux espaces séparés de Fred et 
de Ginger. La caméra descend de Fred et de ses pieds vers le sol de sa chambre (ou, si nous voulons, le plafond de la chambre de Ginger), traverse ce plancher et s'arrête sur la femme dans son lit. A part les connotations plus ou moins évidentes de ce rattachement du corps (sonore) masculin dansant, au corps muet féminin allongé, qui évoque le sujet principal de notre comédie musicale, j'aimerais diriger l'attention sur un autre aspect de cette scène, à savoir sur la qualité médiatique et métaphorique que gagne l'espace fictif du film. L'hôtel s'enrichit de la qualité d'un instrument de percussion, les murs constituant les parties solides et le sol/plafond, le diaphragme ébranlé par les jambes et les pieds des protagonistes. Dans cette perspective, l'espace fictif du film devient donc une sorte d'instrument de percussion que les actions et les corps des protagonistes font résonner. (Fred Astaire traite littéralement de ses pieds non seulement le plancher de cette chambre, mais aussi son mobilier et les murs.) Ce qui me semble une jolie métaphore de l'aspect médiatique de l'espace filmique de la comédie musicale.

\section{- Isn't This a Lovely Day To Be CaUght in the Rain? - INTERTEXTUALITÉ ET INTERMÉDIALITÉ}

Avant de conclure, encore une petite remarque sur le caractère intermédiatique de la scène Isn't This a Lovely Day To Be Caught in the Rain?: elle me semble constituer un bon exemple du labyrinthe médiatique de notre film. Dans ce qui suit, je vais me limiter à énumérer quelques structures principales qui y sont inclues et imbriquées. Pour commencer, c'est le caractère presque littéraire et pictural de ce lieu qui évoque - malgré la pluie - l'idée traditionnelle de l'arcade, du locus amoenus ou du hortus conclusus, du lieu paradisiaque, où la rencontre clandestine et l'aveu des amants peuvent avoir lieu. C'est un lieu séparé du monde ordinaire. Mais ce pavillon possède naturellement et nécessairement aussi les qualités d'une scène théâtrale où la danse et la chanson peuvent être re-présentées. Ce lieu et les actions des protagonistes sont construits sur la base de plusieurs éléments intertextuels ${ }^{15}$ et intermédiaux. Des concepts de la littérature, de la peinture, du théâtre, de l'opérette, pour ne nommer que les plus évidents, sont organisés dans le cadre médiati- 
que et générique de la comédie musicale. Ces médias et ces concepts se trouvent re-codifiés, utilisés selon les besoins du médium "film" et intégrés dans un nouveau contexte médiatique où ils gagnent une nouvelle qualité et une nouvelle fonction.

\section{Un mot pour conclure}

Cette petite analyse de Top Hat m'a mené à des conclusions provisoires. Dans la perspective de la médialité ou de l'intermédialité du film, cette comédie musicale se présente comme un kaléidoscope médiatique dont les interférences et les ruptures médiatiques jouent un rôle considérable en ce qui concerne la fascination qu'exerce ce film sur son spectateur. Même si, pour un spectateur européen, plusieurs dimensions de la lecture de ce film ne sont pas facilement accessibles (il s'agit de lectures qui ne peuvent être effectuées que sur un fond de mentalité historique américaine avec ses racines puritaines), il demeure toujours la possibilité de savourer les structures intermédiatiques de ce genre hybride qui inclut tant de médias avec ses concepts correspondants, dans son jeu de re-présentation filmique. Le jeu intermédiatique de Top Hat peut se produire sous forme d'une citation directe, mais aussi sous d'autres formes plus cachées d'intégration de concepts médiatiques.

Si l'on veut, on pourrait décrire les caractéristiques principales de ce jeu comme des procédures qui veulent faire oublier au spectateur les ruptures entre les différents médias et les différentes structures médiatiques qui se trouvent imbriqués dans le film. Le réalisateur (qui n'existe naturellement que comme "fiction ${ }^{16}$ ") fait tout ce qu'il peut pour que le spectateur ne se rende pas compte qu'il y a un changement perpétuel et une interaction permanente entre différents médias. Souvent, nous ne savons pas quand la chanson ou la danse commencent. Les passages sont imperceptibles et la qualité médiatique de cette comédie musicale se présente conne une sorte d'hybride dont les structures sont synchronisées dans le temps, ou plutôt - pour me référer aux recherches de Rick Altman - fondues dans un nouvel ensemble. Au contraire des concepts postmodernes de l'intermédialité, où c'est au spectateur de constituer une nouvelle "unité intermédiatique", le jeu intermédiatique de Top Hat a 
donc tendance à envisager une fusion aussi grande que possible dans l'acte de production. Et la fascination du film est contenue - selon cet argument - dans la jouissance de cette fusion sans ruptures abruptes de structures et concepts médiatiques différents.

En ce qui concerne les implications de cette approche proposée pour la théorie et l'histoire des genres et du film en général, il me semble évident que la théorie et l'histoire devront se réorienter en incluant dans leur cadre analytique la question du développement des relations et des jeux intermédiatiques dans le médium "film». Le film n'existe pas comme monolithe médiatique et - bien sûr - la comédie musicale est un bon exemple de cette thèse. Dans cette perspective, de nombreuses recherches restent à faire.

\section{Université d'Amsterdam}

\section{NOTES}

1 Je tiens à remercier vivement $\mathrm{M}^{\mathrm{me}}$ Véronique Meyer, de Mannheim, pour son travail et son dévouement dans la traduction de cet article. Larticle est une version raccourcie d'une conférence que j'ai présentée lors du colloque international XI Convegno internazionale di studi sul cinema e gli audiovisiviu qui fut organisé par Francesco Casetti en juillet 1992 à l'Université d'Urbino.

2 Voir Rick Altman, The American Film Musical (Bloomington/London: Indiana University Press, 1987, p. 171 seq.).

3 Voir l'analyse très lucide de Rick Altman, toujours dans The American Film Musical (Bloomington/London: Indiana University Press, 1987, p. 171 seq.).

4 Avec Jean M. Peters, Communicatie van teken tot medium, Leuven, 1985 (p. 3), je rappelle ici le caractère codé et textuel de chaque "médium ".

5 Par rapport à l'intégration de Lyotard dans une théorie de l'intermédialité, voir Karl Prümm, "Multimedialität und Intermedialität. Eine Skizze», Theaterzeitschrift, n 22 (1987-88) p. 93-103.

6 En ce qui concerne ce concept, voir J. E. Müller (direction), "Texte et médialité", Mana, 7 (1987) et J.E.M., "Der inter-mediale Fall des Hauses "Usher": Literatur, Fotografie und Musik - zu einer Bildreihe von Elisabeth Deiters", dans E. W. B. Hess-Lüttich, R. Posner (direction), Code-Wechsel. Texte im Medienvergleich, Opladen : Westdeutscher Verlag, 1990, p. 127-150; ou J. E. M., "Das mediale Labyrinth des Films. Helvio Soto: La triple mort du troisième personnagen, dans J. E. Müller et M. Vorauer (direction), Blick-Wechsel. Tendenzen im Spielfilm der 70-er und 80-er Jahre, Münster: Nodus Publikationen, 1992, p. 201-212.

7 En ce qui concerne les implications de cette position pour l'histoire des médias, je renvoie à mon article, "Intermedialitat als Provokation der Medienwissenschaft", dans Eikon. Internationale Zeitschrift für Photographie \& Medienkunst, H. 4 (hiver 1992) p. 32-41. Patrice Pavis a analysé sous un point de vue sémiotique les interféren- 
ces du théâtre avec les autres médias, dans "Le théâtre et les médias: spécificités et interférences ", dans André Helbo (dir.), Théatre. Mode d'approche. Bruxelles: Klincksieck, 1987, p. 33-63.

8 Ici je suis une argumentation que Jacques Aumont a suggérée pour l'analyse des relations entre peinture et cinéma, dans L'Eil interminable (Paris: Séguier, 1989).

9 Altman a démontré le rôle mythologique de la "radio " dans l'histoire du cinéma au moment de son intervention au colloque XI Convegno internazionale di studi sul cinema e gli audiovisivi, à Urbino, juillet 1992. Le concept médiatique de la radio était, pendant les années vingt (et au début des années trente), très ouvert en comparaison de nos idées actuelles sur ce médium. Derrière le terme Radio Pictures se cachait entre autres, par exemple, l'idée de produire des films qui soient émis par la radio. En ce qui concerne les implications de cette position pour l'histoire des médias, je renvoie à mon article: "Intermedialitat als Provokation der Medienwissenschaft ", dans: Eikon. Internationale Zeitschrift fur Photographie \& Medienkunst, H. 4, 1992, p. 32-41.

10 En ce qui concerne cette influence, je me réfere aussi aux recherches de Karl Prümm, Freie Universität Berlin, sur la première phase du film parlant allemand. En particulier sur sa conférence Early Sound Film in the International Context qu'il a présentée à l'Université d'Amsterdam en juin 1992 (Karl Prümm est en train d'écrire un livre sur ce sujet).

11 En ce qui concerne les implications psychoanalytiques des signes des corps de Fred Astaire et Ginger Rogers, je renvoie à l'article de Cameron D. F. Tolton, "L'CEdipe et la danse: vers une lecture psychoanalytique de Top Hat de Mark Sandrich", Travaux du Cercle méthodologique, n 6 (1979), Department of French, University of Toronto.

12 Christian Metz nous rappelle dans son dernier livre, L'Énonciation impersonnelle ou le site du film (Paris: Méridiens Klincksieck, 1991), que pour pouvoir parler de l'"autoréflexivité" filmique, il ne suffit pas qu'un film nous donne seulement à voir des appareils ou nous "montre le dispositif ". Pour produire un effet d'autoréflexivité, il est nécessaire que le film fasse plus que cela; les éléments nommés doivent entrer dans des jeux complexes (p. 87 et seq.).

13 Voir Rick Altman, The American Film Musical (Bloomington/London : Indiana University Press, 1987).

14 J'utilise ici le texte de la traduction française (Paris: Armand Colin, 1992).

15 En ce qui concerne mon utilisation de la notion de l' "intertextualité", je renvoie ici à l'article suivant de Charles Grivel, "Serien textueller Perzeption. Eine Skizze", dans Wolf Schmid, Wolf-Dieter Stempel (direction), Dialog der Texte. Hamburger Koloquium zur Intertextualitat (Wien: Wiener Slawistischer Almanach, 1983, p. 5383).

16 En ce qui concerne le statut du concept d' "auteur ", voir mes remarques dans mon article "Pragmatique historique du film: Nouvelle Vague et conception de l'auteur. La réception d'A bout de souffle aux Pays-Bas ", dans "Cinéma et réception ", Cinémas, vol. 2, n 2-3 (printemps 1992) p. 57-87.

\section{OUVRAGES CITÉS}

Altman, Rick. The American Film Musical. Bloomington/London: Indiana University Press, 1987.

Lyotard, Jean-François. Dérive à partir de Marx et Freud. Paris: U.G.E., 1973. 